\section{An image-based approach for designing and manufacturing craniofacial scaffolds}

\author{
S. J. Hollister, R. A. Levy, T.-M. Chu, J. W. Halloran, S. E. Feinberg: An image- \\ based approach for designing and manufacturing craniofacial scaffolds. Int. J. Oral \\ Maxillofac. Surg. 2000; 29: 67-71. (C) Munksgaard, 2000
}

Abstract. Bone tissue engineering (BTE), which combines biomaterial scaffolds with biologically active factors, holds tremendous promise for reconstructing craniofacial defects. A significant challenge in craniofacial reconstructive BTE applications is the complex patient-specific geometry that must be reconstructed. In this paper, we present an image-based approach for designing and manufacturing patient-specific craniofacial biomaterial scaffolds directly from CT or MRI data. In this approach, voxel density distribution is used to define scaffold topology. The scaffold design topology is created using image processing techniques. This voxel density distribution is then converted to data that can be used to drive a Solid Free-Form Fabrication machine to either directly build the scaffold or build a mold for the scaffold. Several preliminary applications for craniofacial surgery, including a mandibular condyle scaffold, an orbital floor scaffold, and a general mandibular defect scaffold, are illustrated. Finally, we show applications to in vivo models.

\author{
Scott J. Hollister ${ }^{1}$, Richard A. Levy², \\ Tien-Min $\mathrm{Chu}^{3}$, John W. Halloran ${ }^{3}$, \\ Stephen E. Feinberg ${ }^{4}$ \\ ${ }^{1}$ Departments of Biomedical Engineering, \\ Surgery (Orthopaedics) and Mechanical \\ Engineering and Applied Mechanics, The \\ University of Michigan; ${ }^{2}$ Diagnostic Radiology \\ Associates, Saginaw, Ml; ${ }^{3}$ Department of \\ Materials Science and Engineering, The \\ University of Michigan; ${ }^{4}$ Department of Oral \\ and Maxillofacial Surgery, The University of \\ Michigan, Ann Arbor, MI, USA
}

Key words: craniofacial reconstruction biomaterial scaffolds; imaging; tissue engineering.

Accepted for publication 17 July 1999
Craniofacial reconstructive surgery, in the past, has focused on the designing of replacement parts for facial structures with autogenous, allogeneic, xenogeneic or alloplastic materials ${ }^{7}$. With the debut of the field of tissue engineering, this has shifted to the biological components of tissues: the body's cells, and a scaffold or extracellular matrix ${ }^{1}$. The scaffold can provide both mechanical support and instructive information to assist in the adaptation of the construct to the specific area that will be reconstructed. These grafts or constructs can be reconstituted ex vivo, for specific sites.

The scaffold plays a critical role in the initial development of tissue engineering of the graft for facial reconstruc- tion. It must serve as a carrier for cells and growth factors, optimize the functional capability of the cells and factors (internal geometric organization), assist in early mechanical support for the area to be reconstructed and, lastly, impart proper external architecture to assist in maintenance of esthetics of the area to be reconstructed.

In this paper, we present an imagebased approach for designing and manufacturing patient site-specific biomaterial scaffolds with specific internal architecture using an Image Based Engineering (IBE) design method combined with a Solid Free-Form Fabrication (SFF) manufacturing approach. The process is demonstrated by designing and manufacturing craniofacial structures of interest for reconstruction, including an orbital floor, a mandibular defect and a mandibular condyle.

\section{Material and methods}

Our overall method (Fig. 1) for designing patient-specific scaffolds begins with either Computed Tomography (CT) or Magnetic Resonance Image (MRI) data on the specific craniofacial defect to be reconstructed.

First, the commercial software PV-Wave (Visual Numerics Inc., Boulder, CO, USA) is used to read in the CT data of the area or defect to be reconstructed on the patient. The defect region is then outlined and, by inverting the contrast of the image, a three-dimensional (3-D) image of the defect or region can be generated (Fig. 2). The image of the reconstruction area or defect specifies the contour design of the implant in concert with 
User selects defect region for reconstruction $\downarrow$

Defect Image is created to specify external shape

$$
\downarrow
$$

Internal pore architecture/geometry image is created

$$
\downarrow
$$

Boolean Combination of Defect Image and Architecture Image to create Scaffold Image

$$
\downarrow
$$

Scaffold Image surface extracted to create .stl data$$
\downarrow
$$

.stl data sent to SFF machine

$$
\downarrow
$$

SFF machine manufactures scaffold from biomaterial

Fig. 1. Outline of image-based approach for scaffold design and manufacture.

the adjacent remaining tissue. The same design method can also be used to restore the surface profile of the patient when a change in the patient's profile is associated with a defect. This user interactive process creates the second set of data for the design method, which we denote as the defect image.

The second step in scaffold design is to create the internal pore architecture. The internal pore architecture will determine crucial factors for tissue regeneration including nutrient diffusion, cell and tissue infiltration and mechanical behavior. Although internal architecture must be selected carefully since it can greatly influence matrix deposition ${ }^{6}$, optimizing internal architecture will require extensive in vitro and in vivo experiments. Our purpose in this paper is not to present the optimal parameters, since they are not known, but to present several image-based topology design methods that can create a wide variety of internal architecture designs. Indeed, creating a wide variety of designs is necessary as input to experimentation for determining the optimal parameters.

The idea behind image-based topology design is simply to set voxels within an image design cube to either 0 for no material or 1 for material. We then repeat the basic cube as many times as possible to create the architecture image. A general program was created

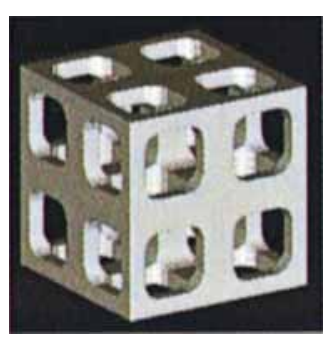

Fig. 2. A general architecture image with pores running in three orthogonal directions. to build up an internal architecture by repeating a unit structure. Using geometric mathematical formulas, images can be created of cylinders, spheres and other similar entities. Using this approach, interior architectural channels are created to allow for cell infiltration and tissue ingrowth (Fig. $3)$.

The above method creates a regular pore structure. Image-based topology design can also be used to create random porous structures. One way is to run randomly set voxels to 0 or 1 using a random number generator. A second method to create pores is by adjusting thresholds of the original defect image. The general histogram of a skeletal CT image is shown in Fig. 4. By setting all voxel densities outside of a narrow threshold range to zero, all of the trabecular bone and also random pores within the cortical bone are created. Using this technique, the pore size will be determined by the image resolution as $\mathrm{mm} / \mathrm{voxel}$
Steps 2 and 3 (see Fig. 1) produce two separate datasets: 1) the defect image, which is the anatomic location where the structure will be placed, and 2) the architecture image, which is an image of the internal architectural pore structure. At this point, using image processing software and setting the solid and void voxel densities in the defect image, the interior architecture image and void spaces to different values, we can combine the defect image with the architecture image. This is done by assigning the void space in both images a density of 0 , the solid portions of the defect image a density of 250 , and the solid portions of the architecture image a density of 200. These different densities allow us to use Boolean operations to combine the pixel values. The result is shown in Fig. 3 .

Once steps 1-4 (Fig. 1) are completed, we now have a digital image representation of the scaffold design. However, to manufacture the scaffold using Solid Free-Form Fabrication (SFF) techniques, it is necessary to

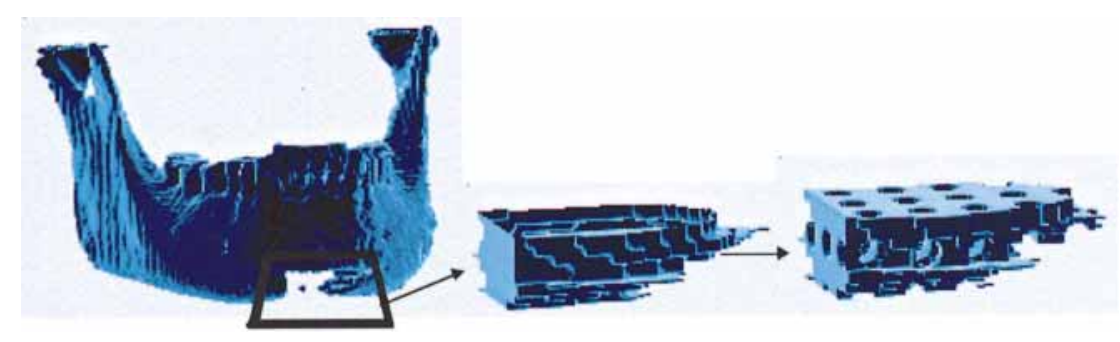

Fig. 3. Scaffold architecture design process applied to mandibular defect. (a) 3-D rendering of original CT scan with defect. (b) Defect image created after user selects defect geometry. (c) Final scaffold image after Boolean combination of defect image and architecture image.

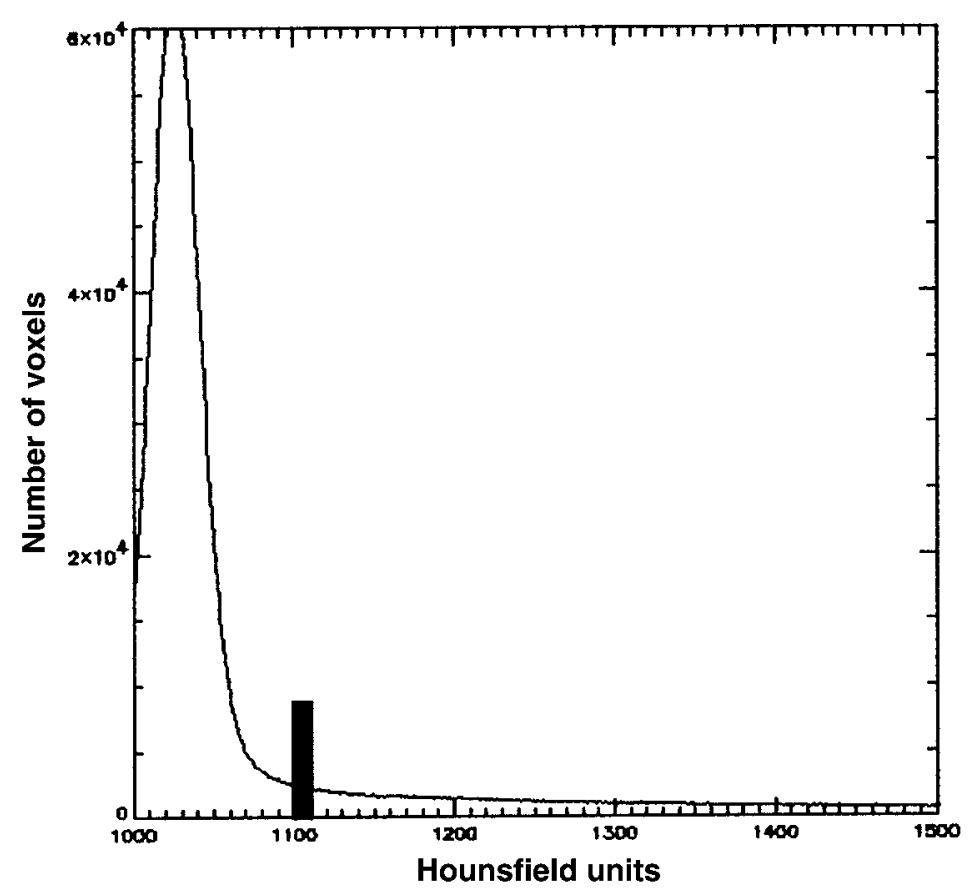

Fig. 4. Example of histogram of CT image for an orbit. Only those densities within the black rectangular box are retained. All others are set to zero. 

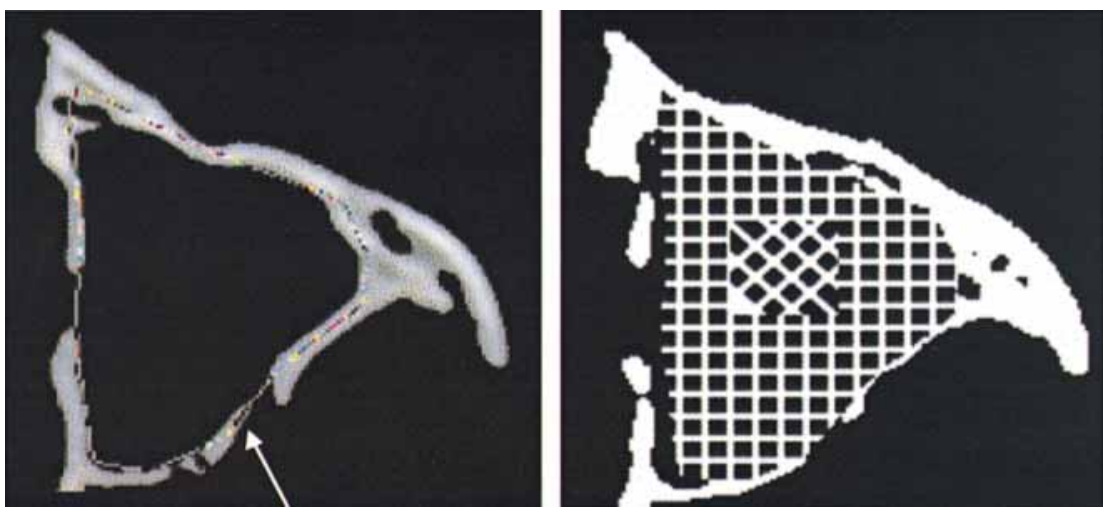

Fig. 5. (a) User selects an outline of the skeletal defect on the image to create the defect image. (b) A porous architecture is then substituted into the defect image to create an internal scaffold architecture within the skeletal defect.

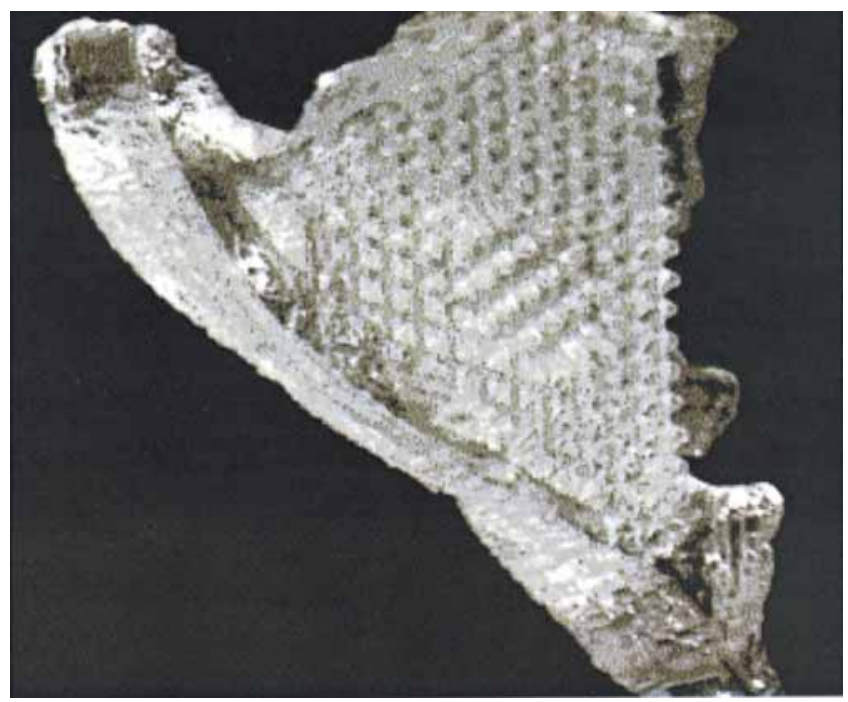

Fig. 6. Final orbital floor scaffold manufactured out of epoxy from the design in Fig. 3.

represent the structure in a data format known as stereolithography or .stl, which defines the surface as connected triangles. Therefore, in step 5, an isosurface density is extracted to produce a triangular facet representation of the surface (Voxelcon 2.0, Voxel Computing Inc., Ann Arbor, MI, USA). This process is automated and produces an ASCII text file with vertices and normals of the triangular facets.

A particular form of SFF called Stereolithography (SLA; 3-D Systems, Valencia, CA, USA) was used to manufacture the scaffolds. In this technique, a computer-driven ultraviolet laser is used to photopolymerize layers of liquid resin. Following photopolymerization of an individual layer, a fresh layer of liquid resin is applied and photopolymerized. The entire process is repeated to build a complex 3-D object from the resin. Recently, Chu, Halloran and colleagues ${ }^{2,3}$ have devised two different techniques to create Hydroxyapatite (HA) structures with SLA. The first is a casting technique in which the inverse image of the 3-D scaffold is generated and a HA slurry is cast into the mold ${ }^{4}$ The second is a direct technique in which HA particles plus acrylate are used in the SLA machine instead of the standard resin. The 3$\mathrm{D}$ scaffold is then built using the layer by layer process. In both cases, the final HA scaffold is sintered to burn out the resin or acrylate and to increase the strength of the ceramic. This gives us the ability to now design and manufacture biomaterial scaffolds that could be implanted within the body ${ }^{5}$.

The purpose of this study was to demonstrate efficacy of the image-based design and manufacturing approaches to construct scaffolds for craniofacial reconstruction. The approach is demonstrated by constructing scaffolds for mandible, orbital floor and condyle reconstruction with both regular pore geometry generated through Boolean operations and with random pore structure constructed through threshold operations. An actual patient CT scan is used as a basis for all constructions. These scaffolds were manufac- tured from epoxy to demonstrate feasibility, although it is possible to manufacture with bioceramics such as hydroxyapatite ${ }^{5}$. Finally, construction of cylindrical HA implants with different internal architectures that have currently been implanted in a Yucatan mini-pig mandible defect model is demonstrated.

\section{Results}

The complete design process (Fig. 1) for the mandibular defect is shown in Fig. 3. First, a defect image is created (Fig. $3 b)$. Second, an internal pore architecture image is substituted within the defect image to create the final scaffold (Fig. 3c).

The image-based design process was also applied to design a porous internal architecture within an orbital floor. The regular pore structure construction began with user selection of the defect that then constituted the defect image (Fig. 5a). A regular porous structure was then substituted for the defect image (Fig. 5b). The final orbital floor scaffold image was converted to .stl stereolithography format and constructed from epoxy on a 3-D Systems SLA 250 machine (Fig. 6).

The second way to create porous internal architecture is through density threshold. In this case, the initial orbit CT scan was threshold such that all densities outside of a narrow range were set to zero to create the porous structure shown in Fig. 7. At this point, the remaining structure size was too small to build on the SLA machine. The boundary pixels were then enlarged using dilation image operations to create a boundary that could be built on the SLA machine (Fig. 8). Fig. 9 shows the final orbital floor constructed from epoxy on the SLA machine.

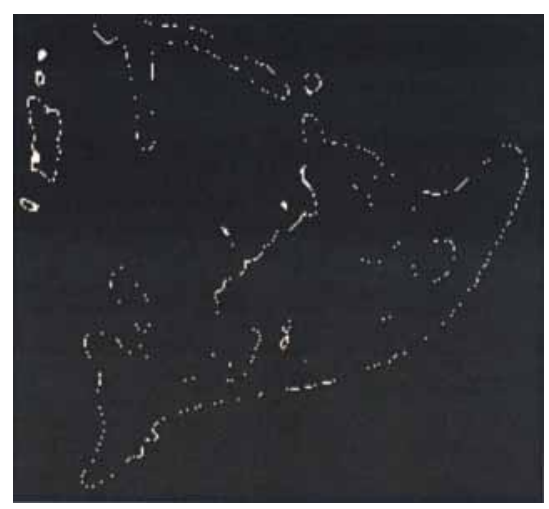

Fig. 7. Outline of the porous image created by selective thresholds. The white dots are the porous structure of the orbital floor. 


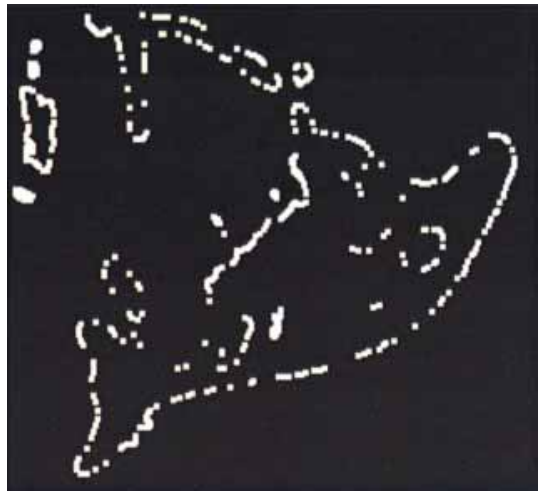

Fig. 8. Same slice as in Fig. 7 with increased boundary thickness following dilation operation.

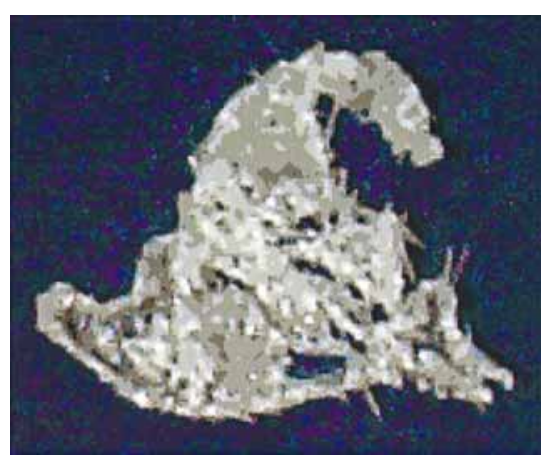

Fig. 9. Epoxy model manufactured from porous orbit scaffold created by threshold and dilation operations.

We have also constructed a scaffold to replace the lateral aspect of a Yucatan mini-pig temporomandibular joint (TMJ) condyle. The mini-pig mandible was scanned at a resolution of 0.488 $\mathrm{mm} /$ voxel. The condylar region was then isolated from the scan and a user selected the lateral aspect for reconstruction on a slice by slice basis using a DEFine Region Of Interest (DEFROI) tool within PV-Wave. Indices of selected voxels were masked within a 3-D array and the internal channel architecture array voxels were substituted for the masked voxels. Boolean intersection operations were performed to create a hole for screw fixation. A rendered view of the designed and manufactured scaffolds is shown in Fig. 10.

Finally, we are beginning to test scaffolds manufactured from HA in vivo. Scaffold architectures created using the IBE procedure were created in $8 \mathrm{~mm}$ cylindrical shapes. These molds were then cast with a HA slurry solution and sintered. An example of the designed and manufactured cylinders along with the in vivo implantation is shown in Fig. 11.

\section{Discussion}

An image-based design and manufacturing approach has been demonstrated that can be used to create patient-specific scaffolds for tissue engineering of craniofacial defects. This approach utilizes currently available modes of imaging, software image processing and image-based design software, and solid free-form fabrication techniques. Using this method, it is possible to create very complex 3-D geometry to fit defects. It is also possible to create a wide range of internal pore architectures to optimize cell tissue infiltration and to tailor mechanical properties in mechanically loaded regions. Although primarily concerned with methods for designing scaffolds, the current techniques can easily be incorporated into surgical planning protocols. The total turnaround time for this process, once the image has been obtained, is typically 24 hours. Of course, this turn-around time could be even shorter with dedicated computing and manufacturing equipment.

The major advantage of this approach is the ability to create a patient anatomic-specific scaffold with a predetermined internal architecture. This approach requires a $\mathrm{CT}$ or MRI scan, which for most craniofacial surgical reconstruction cases is routinely obtained. The computing for this approach can be done on a standard Personal Computer. Using an image data structure, the complete process allows very complex anatomic designs to be created rapidly. These designs can also contain various $3-\mathrm{D}$ internal pore architectures. Pore architectures are important for allowing cell migration and tissue integration into the scaffold. If traditional CAD techniques were to be used, one would first have to convert image data into CAD geometry representation. Within this CAD geometry representation of an arbitrarily complex 3-D anatomic shape, internal pore ge-
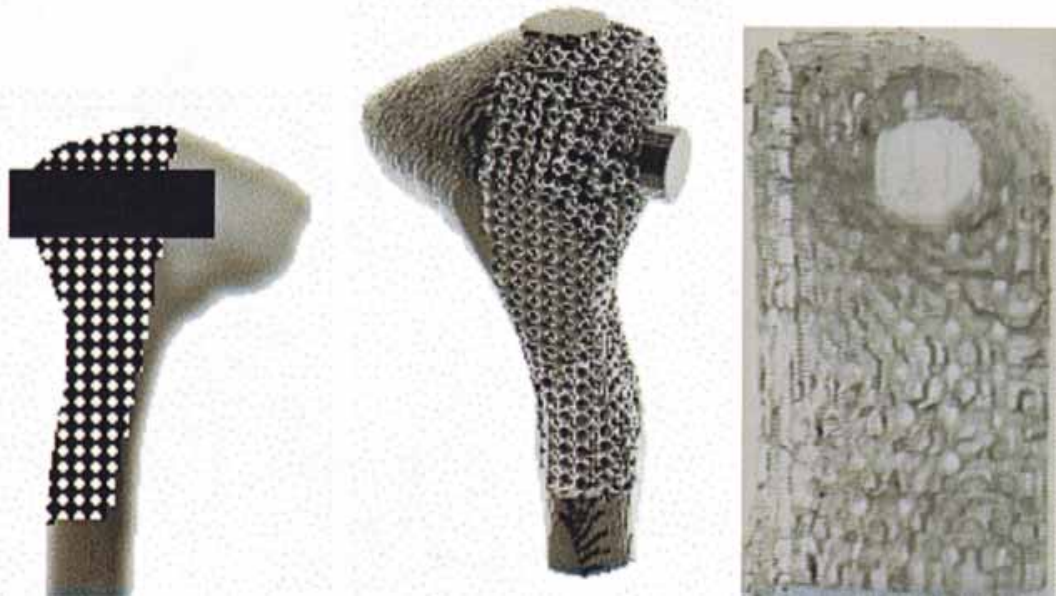

Fig. 10. Comparison of designed and manufactured condyle scaffold. (a) Slice of condyle with scaffold and fixation screw. (b) 3-D rendered version of condyle with scaffold and fixation screw. (c) Example of epoxy mold manufactured using stereolithography.

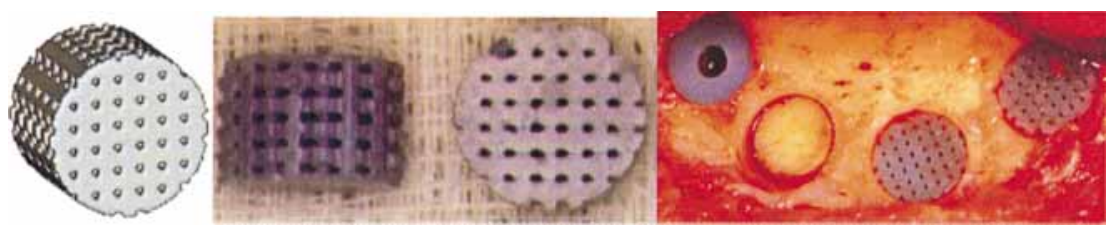

Fig. 11. (a) Image-based scaffold design of cylinder with orthogonal pores. (b) Actual HA scaffold made by casting HA slurry in inverse epoxy mold of scaffold. (c) Surgically implanted scaffolds in mini-pig mandible defect model. 
ometry has to be constructed and interfaced with the anatomic shape. Such operations can take even experienced CAD software users a few days to complete. Thus, the image-based approach to designing scaffolds provides for a much faster creation of scaffold designs. The mandible design illustrated in this paper was created in less than 30 minutes.

In conclusion, we have presented a general image-based design and manufacturing approach for constructing anatomic scaffolds for craniofacial reconstruction. This technique allows rapid design and construction of complex anatomic shapes with complete control over external shape and internal pore architecture. These techniques can be combined with biomaterial processing ${ }^{5}$ to directly construct scaffolds for craniofacial reconstruction. This avenue is currently being pursued to construct scaffolds for reconstructing the temporomandibular joint.

\section{References}

1. BeLl E. Strategy for the selection of scaffolds for tissue engineering. Tissue Engineering 1995: 1: 163-79.

2. Chu T-M, Halloran JW. Hydroxyapatite for implant fabrication by stereolithography. In: Ghosh A, Hiremath B, BARKS R, eds.: Case studies of ceramic product development, manufacturing, and commercialization. Ceramic Transactions. Vol. 75. Westerville, OH: American Ceramic Soc., 1997: 119-25.

3. Chu T-M, Halloran JW, Wagner WC. Ultraviolet curing of highly loaded hydroxyapatite suspension. In: RUSIN RP, Fishman GS, eds.: Bioceramics: materials and applications. Ceramic Transactions. Vol. 65. Westerville, OH: American Ceramic Soc., 1995: 57-66.

4. Chu T-M, Halloran JW, Hollister SJ, FeINBERG SE. Hydroxyapatite with controlled internal architecture by acrylic polymerization. J Mater Sci Mater Med (submitted).

5. Levy RA, Chu TMG, Halloran JW,
Feinberg SE, Hollister SJ. Computed tomography-generated porous hydroxyapatite orbital floor prosthesis as a prototype bioimplant. Am J Neuroradiol 1997: 18: $1522-5$.

6. Tsuruga E, Takita H, Itoh H, WakiSAKA Y, KubOKi Y. Pore size of porous hydroxyapatite as the cell-substratum controls BMP-induced osteogenesis. J Biochem 1997: 1211: 317-24.

7. Yaszemski MJ, Payne RG, Hayes WC, LANGer R, Mikos AG. Evolution of bone transplantation: molecular, cellular and tissue strategies to engineer bone. Biomaterials 1996: 17: 175-85.

Address:

Scott J. Hollister, PhD

Department of Biomedical Engineering

Rm 3304 GG Brown Bldg.

2350 Hayward

Ann Arbor, MI 48109-2125

USA

Tel: +1 (734) 6479962

e-mail: scottho@umich.edu 